\title{
Cuestionar y problematizar la propia práctica: Investigación Acción Transformadora en los procesos de desarrollo profesional docente
}

\section{Question and problematize own practice: Transformative Action Research in teacher professional development processes}

\author{
Ignacio Figueroa Céspedes ${ }^{1}$ \\ María Paz Gómez Montecinos \\ Centro de Desarrollo Cognitivo, Santiago, Chile \\ (Rec: septiembre de 2015 - Acept: noviembre de 2015)
}

\begin{abstract}
Resumen
El profesorado juega un rol fundamental en la construcción de nuevas alternativas para entender y mejorar los procesos de enseñanza y aprendizaje. Las reflexiones que hagan al respecto se tornan fundamentales para afrontar cambios en un sistema educativo altamente segregado, homogeneizador y desigual. En este sentido, el desarrollo profesional docente se abre como una herramienta de cambio en la medida que dichos espacios propicien el análisis y mejora de la propia práctica docente, fomentando en los y las docentes una mirada crítica, reflexiva y transformadora. Este trabajo propone articular los aportes de la Investigación-Acción Transformadora (IAT), entendiendo como base la creencia en el potencial del docente para fomentar procesos sociales de construcción de aprendizajes en un contexto de diversidad y heterogeneidad.
\end{abstract}

Palabras clave: Investigación Acción Transformadora, desarrollo profesional docente, profesionalidad docente.

\begin{abstract}
Teachers have a key role in building new ways to understand and improve the processes of teaching and learning. Reflections about it become essential to address changes in a highly segregated, homogenizing and unequal education system. In this sense, teacher professional development becomes a tool for change, insofar as such programs would encourage the analysis and improvement of teaching practice from a critical, reflective and transformational view. This paper intends to articulate the contributions of the Transformative Action Research (TAR) grounded on teachers' potential to generate changes in the education system, even beyond its own barriers, to promote social processes within the construction of learnings in a context of diversity and heterogeneity.
\end{abstract}

Keywords: Transformative Action Research, teacher professional development, teaching professionalism. 


\section{Introducción}

\section{“...Y si no existen preguntas, es difícil que se busquen respuestas..."}

\section{(M.A. Santos Guerra)}

Iniciar procesos de transformación y cambio implica situarse en territorios desconocidos e inciertos, para lo cual resulta imperioso contar con convicciones claras respecto a lo que nos moviliza y nos da sentido en el trabajo educativo. Dichas convicciones contienen información relevante acerca de lo que debemos hacer para propiciar nuevas estructuras, relaciones y entornos que desde procesos construidos colaborativamente generen propuestas de re-significación y el enriquecimiento de la propia práctica docente.

De acuerdo a Beca (2005 en Venegas \& Campos, 2010), los procesos formativos se distancian de la realidad del aula, dado que las dificultades de trabajo reales o la diversidad de los estudiantes no son consideradas en los espacios académicos o de capacitación. La distinción teoría-práctica, herencia de los enfoques positivistas, aún está muy presente en nuestro sistema educativo.

Estos tránsitos nos llevan a cuestionar lo que hoy en día ocurre con nuestra educación y, en particular, con el desarrollo profesional docente, en donde seguimos observando dinámicas que contribuyen a la perpetuación de un modelo de sociedad neoliberal, altamente jerarquizado, individualista, aislado y reproductivo. Cursos de capacitación sobre didáctica de las matemáticas al servicio de las evaluaciones estandarizadas, métodos de evaluación cada vez más eficientes, rendición de cuentas (accountability) centrada en resultados académicos, son muestra de ello. Así pues, ¿cómo favorecer propuestas que ofrezcan una alternativa a este modelo y que emerjan desde la propia experiencia docente?

$\mathrm{El}$ aula configura un potente espacio interaccional, sin embargo, la raigambre tradicional la traduce en relaciones que se forman bajo la idea de construcción, pero caracterizada por la jerarquización y unidireccionalidad de dichos procesos. El docente reconoce teorías acerca del aprendizaje, sus características y estudios que las avalan y argumentan acerca de las variables implicadas. No obstante, cuando se pone en práctica la teoría aprendida, se observan importantes brechas. Entonces ¿qué procesos podrían favorecer una apropiación contextualizada, que posibilite la integración entre teoría y práctica? ¿Cuál es el aporte de la problematización de la realidad educativa para generar transformaciones educativas? Estas preguntas nos orientan hacia la tesis de este artículo: la factibilidad de abordar la práctica desde la reflexión docente, propiciando una mirada crítica y contextualizada del aprendizaje. A partir de la investigación de la propia acción docente, emergen nuevas rutas para dotar de un sentido transformador al quehacer profesional en el contexto de las instituciones escolares.

En relación a esta materia, Imbernón (2001) plantea que el desarrollo profesional docente debiera atender a cinco ejes fundamentales de transiciones paradigmáticas que configuran una agenda transformacional: 1 . Del problema específico a la situación problemática, entendida en contexto; 2 . De la individualidad al trabajo colaborativo; 3 . Del objeto de formación al sujeto de formación; 4. De la formación aislada a la formación comunitaria y; 5 . De la actualización teórica a la creación de espacios de participación.

En este marco, la formación continua de docentes como vehículo de desarrollo profesional se constituye en una instancia llena de posibilidades, resultando clave tanto en sus fundamentos como en sus acciones. Aporta un excelente espacio para invitar a los y las docentes a mirar multidimensionalmente los elementos contextuales y mirarse a sí mismos. De este modo, se favorece la transformación de una manera activa, generando nuevas modalidades de desarrollo profesional cada vez más colectivas y contextualizadas.

El presente artículo busca sistematizar desde una perspectiva reflexiva algunos fundamentos que permiten relevar a la Investigación Acción Transformadora como un dispositivo capaz de articular procesos sociales y personales, propiciando la formación de un rol docente dinamizador de procesos de enseñanza y desarrollo, en un contexto de diversificación curricular. En este sentido, esta propuesta aparece como una oportunidad para generar procesos de transformación de la realidad educativa a partir de experiencias de aprendizaje basadas en la reflexión, el diálogo y la participación.

\section{Antecedentes contextuales}

Chile presenta enormes brechas de desigualdad social, la cual se experimenta en el ámbito educativo, resultando ser una situación que afecta todos los niveles de acción docente. Al respecto, García-Huidobro (2007) destaca el carácter segregador que tiene Chile, específicamente, en cuanto a su sistema educativo: 
El sistema educacional chileno posee todas las limitaciones de un sistema altamente segmentado: no ofrece a los alumnos un entorno óptimo de aprendizaje y perjudica la educación ciudadana, ya que pierde su capacidad de ofrecer una experiencia igualitaria de base que refuerce la igualdad de los ciudadanos y sirva de referente a la universalidad de lo estatal. (p. 80)

El panorama educativo es altamente preocupante: "Un sistema educacional cuya fisonomía expresa, casi con la fidelidad de un retratista, a los sectores sociales del que provienen los alumnos, es un sistema que, además de injusto, deteriora el espacio de lo público" (Peña, 2002 como se citó en García-Huidobro, 2007, p. 81). Al respecto el mismo autor concluye:

Las consecuencias sociales de este panorama escolar son fuertes: las escuelas chilenas como vimos son incapaces de hacer experimentar, en la vida escolar cotidiana, la realidad de un país plural, diferenciado, y entregan, por tanto, una imagen distorsionada de la realidad. (p. 81)

En este contexto emergen las nuevas políticas de inclusión escolar. En particular la Ley 20.845, que regula la admisión de los y las estudiantes, elimina el financiamiento compartido y prohíbe el lucro en establecimientos educacionales que reciben aportes del Estado (Ministerio de Educación [MINEDUC], 2015). Esta iniciativa busca terminar con la discriminación arbitraria, favoreciendo la accesibilidad universal y la gratuidad, y el cambio de la estructura organizativa y financiera de las instituciones que reciben aporte estatal. Esto, unido a la discusión sobre la carrera profesional docente, decretos de diversificación curricular y proyecto de ley sobre el fortalecimiento de la educación pública, nos sitúa en un contexto de problematización de la estructura de nuestra educación.

Este contexto de reforma y de discusión educativa, es propicio para efectos de este artículo, ya que asume al docente como parte de un sistema con características particulares que, de una u otra manera, influye en su pensar y actuar específico. De una manera u otra, el docente está involucrado en el sistema educativo, en sus inequidades y lógicas sistémicas. Es así como surge el debate acerca de la relevancia del rol docente en los procesos de enseñanza y aprendizaje. Diversos estudios hacen un intento por definir el peso de las variables que determinan el impacto que, finalmente, tiene el profesorado en el aprendizaje y el desarrollo de los estudiantes.

En cuanto a lo que sucede dentro del aula, el Centro de Medición de la Universidad Católica (Herrera, 2015) analizó los resultados de la Evaluación Docente 2013, donde emergen desempeños débiles, en particular, en la dimensión interacción pedagógica vinculada con la retroalimentación otorgada por el profesorado hacia sus estudiantes. En este caso, el $81 \%$ de los maestros alcanzó un nivel básico e insatisfactorio. Para Zapata (como se citó en Herrera, 2015) los y las docentes no retroalimentan los aprendizajes a partir de los resultados de sus evaluaciones. En este sentido, Martinic y Vergara (2007) investigan la gestión del tiempo e interacciones entre docentes y estudiantes en la sala de clases, encontrando que el $73 \%$ de las intervenciones en el contexto de la clase son del profesor, exclusivamente, y que el desarrollo de la clase orientado a un proceso mayoritariamente instruccional ocupa un $89 \%$ del tiempo (Villalta, 2000 como se citó en Martinic \& Vergara, 2007). De acuerdo a estas investigaciones, se podría colegir que nuestros docentes, hoy en día, están focalizados en responder instrumentalmente a las exigencias del sistema (hacer clases sigue siendo "pasar la materia") y no en fomentar el desarrollo cognitivo integral.

El informe "¿Quién dijo que no se puede? Escuelas efectivas en sectores de pobreza" (Fondo de las Naciones Unidas para la Infancia [UNICEF], 2004), desde un enfoque de efectividad escolar, aporta elementos al debate planteado y concluye falta de coherencia entre lo que ocurre en la sala de clases y el proyecto educativo, aportando visiones respecto a la necesidad de contar con un proceso de formación docente permanente y en terreno.

Estos datos resultan interesantes, sobre todo si se desea plantear la necesidad de transformar prácticas desde la problematización de la realidad educativa como es el caso de las interacciones pedagógicas que vimos anteriormente. Esto porque, además de los datos empíricos con respecto a las prácticas de aula, es fundamental conocer y reconocer las voces de las/ los docentes al respecto y si efectivamente existe una postura crítica frente a esta problemática.

Un estudio llamado "Proceso de EnseñanzaAprendizaje desde la perspectiva de los profesores en Chile" (Centro de Investigación y Desarrollo de la Educación [CIDE] \& Universidad Alberto Hurtado [UAH], 2012) nos da la posibilidad de conocer las creencias que fundamentan las decisiones que toman 
los y las docentes del país, con el fin de generar procesos de mejora a nivel de formación inicial y continua de los y las docentes. Este objetivo es de suma importancia para abordar nuestra temática de estudio, ya que el desarrollo profesional docente es parte de un proceso de investigación de las necesidades que surgen desde los mismos actores del sistema. Se busca con esta reflexión que los educadores participen y fomenten el diálogo en torno a las problemáticas educativas, poniendo a disposición del aprendizaje los razonamientos particulares de cada persona. Los y las docentes "asumen que la realidad es compleja, pero que en esa complejidad, hay un pequeño espacio para la acción individual. Hay un pequeño espacio para sumergirse en las fisuras del sistema y generar un cambio" (CIDE \& UAH, 2012, p. 80). Desde una perspectiva crítica pero a la vez esperanzadora, el desafío que emerge es propiciar que el profesorado se represente los cambios no sólo como utopías, sino como fuente de inspiración para su propia práctica pedagógica. ¿Será esto posible?

Otro estudio realizado por Darling-Hammond (2003) apunta a las expectativas del profesorado como indicador del rendimiento del/la estudiante. Dicha temática también merece interés si vemos al docente como sujeto histórico que da significado a sus experiencias, en el sentido de que las posibilidades de interacción también dependerán del valor que le asigna el/la docente al proceso co-constructivo con sus estudiantes y los logros de aprendizaje que puede obtener en él. Esto se relaciona directamente con la interrogante respecto a la problematización de la realidad, ya que la trama de sentidos y significados que se tejen en esa interacción van a estar determinadas en gran medida no sólo por las expectativas, sino que por lo que está tras ellas: las creencias de los y las docentes.

Para Pérez Echeverría, Mateos, Scheuer y Martín (2006), el análisis no se centra sólo en describir, sino también en contrastar discurso y acción educativa, afirmando que existen algunas dificultades que son parte del proceso de análisis de creencias organizadas en "Teorías implícitas" en los profesores. Estas teorías tendrían tres componentes fundamentales: en primer lugar, el origen de los procesos de aprendizaje; en segundo lugar, el funcionamiento de estos procesos referidos al ámbito cognitivo y, finalmente, la posible forma de cambio de estas representaciones. El desafío, desde el punto de vista de los autores, no es sólo en función del anhelo de una transformación en las prácticas pedagógicas, sino que también se refiere a favorecer la comprensión que lleve a cambiar las relaciones entre estos componentes y “(...) el conocimiento explícito que mantenemos para esas mismas situaciones” (p. 98).

Por otro lado, Pozo y Torrado (2006) concluyen que las prácticas pedagógicas de los y las docentes distan mucho de ser constructivas (teoría que aparecía mayoritariamente en el discurso del profesorado). "Los profesores, al menos en el caso de este estudio, (...), muchas veces no dicen lo que hacen (¿o tal vez sea que no hacen lo que dicen?)" (p. 226). Lo que interesa destacar del aporte de estos autores es que se abre un camino en la búsqueda de alternativas para que esta dicotomía escénica de nuestros docentes no sea sólo objeto de estudio de expertos, sino que permita, a través de una permanente actitud investigativa, lograr que los propios involucrados visibilicen y problematicen estos procesos para modificar sus prácticas pedagógicas.

Desde un enfoque normativo, el Marco para la Buena Enseñanza (MINEDUC, 2004) aparece como una estrategia para definir ciertas competencias del "buen docente", destacando su relevancia en el proceso de mejora educativa. Desde sus lineamientos se refiere a ciclos del proceso de enseñanza y aprendizaje, y a una serie de dominios caracterizados por criterios que a su vez se componen de descriptores de las acciones que se esperan de un educador. En esta oportunidad se referirá el dominio D, "Responsabilidades profesionales", en el cual se describe el primer criterio: "El profesor reflexiona sistemáticamente sobre su práctica" (p. 15). Este punto tiene como objeto, por una parte, que el profesor evalúe en qué medida los estudiantes alcanzaron los aprendizajes esperados, que analice críticamente su práctica de enseñanza y que la reformule desde el análisis de resultados de aprendizaje de los estudiantes. Por otra parte, también se persigue que el profesor identifique sus propias necesidades de aprendizaje y procure satisfacerlas.

Lo anteriormente mencionado es sumamente trascendente e invita a plantearse si el sistema educativo está fomentando que el educador conozca sus propias necesidades de aprendizaje a partir de sus debilidades y fortalezas. Desde ahí se reconoce la necesidad de establecer una relación entre enseñanza e investigación, pero no de tipo tradicional, referida a la creación de teorías sobre la educación. Se trata, más bien, de concebir a la enseñanza como una actividad autorreflexiva e investigadora en sí misma; así como un fenómeno socio-cultural complejo, socialmente construido, interpretado y llevado a cabo por los propios docentes (Latorre, 2003). 
Es relevante destacar, desde esta perspectiva, que no siempre se concibió lo anterior como posible y que, en primer lugar, se tuvo que llegar a criticar lo que hoy en día aún observamos en nuestros sistemas educativos. Dewey (1993 como se citó en Latorre, 2003) lo describía señalando que los educadores tendían a actuar en forma reactiva, por saltos acríticos, de una técnica a otra. Como alternativa propone que los educadores se movilicen a partir de sus propias ideas.

En esta línea, pasa a ser relevante la concepción de desarrollo humano que tiene el profesorado y su concordancia con las estrategias y experiencias que como educador ofrece en el espacio de interacción educativa. El estudio mencionado previamente en relación con las creencias de los profesores se vincula con lo planteado por Elliott (2000) con respecto a esta dualidad de acción como consecuencia de la pasividad en la indagación. La investigación realizada por el CIDE (2012) dice al respecto:

Las creencias docentes sobre EnseñanzaAprendizaje suelen aludir, en el contexto chileno, a sistemas de cognición tradicionales, de tipo (neo) conductista que, no obstante, coexisten con ideas más ligadas al constructivismo. Explicar esas diferencias es el objetivo de la tarea investigativa. (p. 31)

Esta conclusión con respecto a lo que sucede en cuanto a cómo se concibe el proceso de enseñanza y aprendizaje se complementa con lo que se rescata desde la opinión de directivos y jefes técnicos entrevistados en el estudio:

Se describe que las prácticas se sitúan en la lógica de transmisión y reproducción del conocimiento, con un enfoque más orientado al conductismo, ya que la puesta en práctica de un escenario de enseñanza-aprendizaje constructivista, exige una serie de condiciones que no están presentes en el aula y son complejas de instalar. (p. 84)

No deja de sorprender entonces que "el constructivismo" quede reflejado como una opción irreal, lejana y poco probable de concretar. Ahora bien, los procesos subyacentes a estas creencias están basados en un pensamiento que afirma que los y las docentes van aprendiendo y mejorando su quehacer en la medida que, en un proceso competitivo, reciben premios o castigos como incentivos por desempeño (OPECH, 2007). Si el paradigma de desarrollo profesional docente fomenta hoy en día premios y castigos como incentivos de mejora, ¿como hacer que la acción docente en sí misma, sea reflejo de acciones docentes innovadoras que impulsen procesos educativos cada vez más pertinentes y desarrolladores?

Debido a esto, es necesario poner bajo examen las políticas educativas de desarrollo profesional docente, en particular, las que tienen que ver con la formación continua, porque implican asumir que los educadores se forman en la teoría y en la práctica. Los educadores no nacen, sino que se hacen, y dado que se hacen, también se deshacen y se rehacen. Por lo tanto, es prudente ver y situar en la práctica aquellos procesos que favorecen la apropiación de un sentido concordante tanto en sus concepciones como en sus posiciones y acciones. Se dirá entonces que no existirá desarrollo curricular sin un desarrollo del profesor como primer paso (Stenhouse como se citó en Latorre, 2003). En consecuencia, este artículo postula que no hay desarrollo de los estudiantes sin un desarrollo crítico del profesorado en tanto individuo y en tanto colectivo.

\section{Metodología}

Este artículo se construyó desde el paradigma cualitativo de investigación social, el cual destaca la comprensión como principal objeto de análisis. Se utilizó la metodología del self-study como vehículo para dar cuenta de las reflexiones emanadas de la implementación de programas de formación continua con inspiración en la investigación-acción, desarrollando roles de coordinación docente en el equipo del Centro de Desarrollo Cognitivo de la Universidad Diego Portales. En este contexto, se utilizan dichas experiencias como un recurso para problematizar el "ser" en su relación con situaciones prácticas con la meta de redefinir sus creencias o práctica (Feldman, 2002 como se citó en Samaras \& Freese, 2009). El propósito fue el desarrollo de un marco conceptual a partir de la reflexión y discusión teórica acerca de la relación entre la investigación-acción y el desarrollo profesional docente en el contexto chileno; todo ello desde una mirada crítica. Se recogieron notas reflexivas y diarios de campo de los autores, los que se analizaron permitiendo dialogar con los marcos teóricos abordados.

Para efectos de este artículo se trabajó con un total de 30 manuscritos recopilados entre septiembre de 2012 y diciembre de 2014 siendo considerados como material a analizar. Recurrimos a las orientaciones de la Teoría Fundamentada (Strauss \& Corbin, 2002), 
realizando una codificación simple del material empírico, proceso que nos permitió configurar los ejes analíticos que a continuación se presentan en forma de resultados.

\section{Resultados}

Investigación Acción Transformadora (IAT): democratizar la investigación, valorar la profesión docente

La investigación-acción es definida por Elliott (1991 en Hernández, Fernández \& Baptista, 2008) como el estudio de una situación social con miras a mejorar la calidad de la acción dentro de ella. Si bien la forma concreta que adopta una investigación-acción varía, en general, sigue una secuencia en espiral que supone: planificación, identificación de hechos, análisis, implementación y evaluación, y así sucesivamente (Lewin en Hernández, et al, 2008). Entre los aportes más significativos de la investigación-acción a la educación podemos destacar las siguientes (Blández, 2000 en Blández, González, López \& Sierra, 2004):

1. Forma un profesorado reflexivo.

2. Permite que los y las docentes investiguen.

3. Aumenta la autoestima profesional.

4. Refuerza la motivación profesional y rompe con la soledad docente.

Desde esta perspectiva, emerge la práctica de la investigación-acción con un doble potencial en términos del desarrollo profesional docente ya que, por una parte, identifica un problema de la práctica educativa proponiendo soluciones pertinentes y contextualizadas y, por otra, alienta la comprensión de los educadores acerca del fenómeno educativo aumentando las posibilidades de "apropiación participativa" (Roggoff, 1997 en Castorina \& Dubrovsky, 2004) y fortaleciendo sus capacidades profesionales.

El cuestionar y problematizar la práctica pedagógica y educativa presupone, según Boggino y Roserkrans (2007), el necesario camino de "democratizar el proceso de investigación" (p. 30), lo que desde la perspectiva de este artículo es fundamental. Que los y las docentes se sientan con la propiedad de abordar y reconocer sus propias prácticas para enriquecerlas implica un proceso de apropiación, el cual los empodera en sus prácticas educativas, recuperando el sentido emancipador de la educación.
La investigación-acción educativa, puede caracterizarse, siguiendo a Elliott (2000), con varios rasgos distintivos, entre ellos:

1. Se centra en el descubrimiento y resolución de los problemas que enfrentan los educadores.

2. Supone la reflexión simultánea sobre los medios y los fines. Los valores educativos se definen como fines mediante las acciones concretas que seleccionan los educadores para su realización.

3. Concibe las actividades de enseñanza como interpretaciones prácticas acerca de los valores. Esto implica razonar sobre la calidad de la enseñanza impartida, reflexionando sobre aquellos valores que definen dicha calidad.

4. Analiza acciones y situaciones sociales experimentadas por los profesores vinculando "problemas cotidianos" con "problemas teóricos" definidos por los investigadores.

5. Busca profundizar la comprensión de un problema, lo cual no supone en sí mismo una respuesta específica a dicho problema.

6. Es recursiva a la hora de identificar y desarrollar acciones que puedan aportar más comprensión.

7. Requiere diálogos libres de trabas, debiendo basarse progresivamente en la autorreflexión de los participantes, promoviéndola activamente.

Como señala Carr y Kemmis (en Latorre, 2003), la investigación-acción crítica está íntimamente comprometida con la transformación de la organización y de la práctica educativa y social. Incorpora las ideas de la teoría sociocrítica, abordando la praxis desde su objetivo principal: la emancipación del profesorado (sus propósitos, prácticas rutinarias y creencias). Es un dispositivo que permite vincular su acción al contexto en donde se desarrolla, ampliando la transformación a otros ambientes. El foco es la modificación de las formas de trabajo pedagógico (constituidas por el discurso, la organización y las relaciones de poder), por lo que trasciende lo técnico-instrumental, para evolucionar hacia un proceso crítico de intervención y reflexión. Involucra indagación y conocimiento, propone un proceso dinámico de acción y cambio, y una visión ética y valórica que se pone al servicio de la comunidad educativa.

El tránsito de un estado presente hacia otro estado futuro se origina desde la agencia de una serie de acciones que realizan las personas que se encuentran en este espacio de cambio; los sujetos involucrados creen, por una parte, en la potencialidad de las circunstancias $\mathrm{y}$, por lo mismo, apoyan el proceso desde una mirada 
problematizadora. De acuerdo a lo anteriormente expuesto: ¿Como potenciar procesos de reflexión colectiva en las distintas experiencias de desarrollo profesional docente? ¿Podrían los educadores generar procesos de "Apropiación Participativa" sobre dispositivos de investigación-acción? ¿Cómo podría esto influir en el análisis y posterior transformación de sus prácticas educativas?

Nuestra visión es que las comunidades docentes serán capaces de desarrollar sus propios recursos pedagógicos en la medida que generen procesos de problematización de su propia práctica, haciendo cotidiano este hacer reflexivo, y que este diálogo «libre de trabas» se abra como una opción del quehacer pedagógico. Pensando en que todo sistema demuestra fisuras identificables, como lo vimos anteriormente, la acción transformadora de un profesorado empoderado, como palanca de cambio, permitirá abrir espacios de rescate del propio potencial y del de sus estudiantes. Así mismo, modificará el curriculum desde la experiencia y recuperará miradas desde el enriquecimiento en la diversidad y la co-agencia con la comunidad.

Es a partir de estas preguntas que los investigadores llegan específicamente al método de la Investigación Acción Transformadora (IAT), la que será entendida como un proceso de investigación participativa con miras a mejorar la calidad y pertinencia de las respuestas pedagógicas, buscando la transformación de las prácticas docentes problemáticas. De acuerdo a lo analizado, se proponen algunas categorías que rescatan el aporte de la IAT a los procesos de desarrollo Profesional, que proponen tensiones conceptuales, a saber:

1. "Usuarios v/s Creadores de Curriculum". Profesionalidad Activa y Reflexiva.

2. "Aislamiento v/s Comunidad". Investigar y construir comunidad.

3. "Competitividad v/s Colaboración". Trabajo Colaborativo y Co-Agencia.

4. "Reproducción $v / s$ Transformación". Nociones de Transformabilidad y Cambio.

5. "Estandarización $v / s$ Diversificación". Diversificación y contextualización del desarrollo profesional docente.

A continuación se describirán dichas categorías, proponiendo relaciones teóricas e implicancias prácticas.

\section{1. "Usuarios v/s Creadores de Curriculum". Profesionalidad Activa y Reflexiva.}

Una educación que promueva la acción reflexiva, comenzando por los propios docentes, es una educación que brinda posibilidades, que se aleja de las miradas homogeneizadoras y actúa transformando el espacio educativo en un espacio de genuino encuentro con el conocimiento, comenzando por el diálogo y la aceptación de las diferencias humanas. En concordancia con el planteamiento de Giroux (1997), entendemos a los profesores como "Intelectuales Transformativos", visión que a su juicio nos "capacita para empezar a repensar y reformar las tradiciones y condiciones que hasta ahora han impedido que los profesores asuman todo su potencial como académicos y profesionales activos y reflexivos" (p. 177).

En la base de este concepto está el objetivo de movilizar al profesorado desde un papel consumista pasivo, identificado con ser "usuario" del currículum, vale decir, un sujeto "aplicador" de estrategias, hacia un educador activo que propicia la indagación, adoptando una posición determinada frente a temáticas que le competen como profesional (Elliott, 2000). Es por ello que el docente en su práctica requiere de la investigación para re-crear el dinamismo del conocimiento y re-vivenciar el aprendizaje (y el curriculum) a partir de la reflexión critica de su misión/visión en la educación (Zuloaga, 2010).

El desarrollo profesional docente es, al mismo tiempo, un deber y un derecho de los y las docentes (Amaya-De Rebolledo \& Amaya, 2010), ya que comporta la responsabilidad de quienes toman decisiones en el ámbito educativo, e implica la búsqueda de estrategias adecuadas de formación continua. Esto conlleva implícita la exigencia de brindar autonomía, dignificando y potenciando la profesión docente, con vistas a mejorar la calidad de nuestra educación.

\section{2. "Aislamiento v/s Comunidad". Investigar y construir comunidad.}

El desarrollo profesional más significativo del docente no se sitúa en una charla, taller, o en una conversación aislada, sino que sucede a través del aprendizaje dialógico y comunitario, ambos resultantes 
en contextos vinculados y pertinentes a su práctica (Molina, 2003). El desarrollo profesional docente debiera tener como objetivo, entonces, no solo el progreso individual, sino la promoción de aprendizajes en entornos comunitarios donde el principal influjo sea generar un grupo cohesivo y, como consecuencia, mejorar individualmente.

El diálogo reflexivo como instrumento de aprendizaje se inscribe en torno a la necesidad de profundizar en experiencias que generen transformaciones a través de la comunicación activa como eje central. Así, la concepción comunicativa planteada por Elboj, Puigdellivol, Soler y Valls (2002) destaca el valor de las interacciones como motor en la construcción de significados en un contexto de diálogo igualitario, buscando comprender al otro, diseñar y planificar acciones que surjan de esta necesidad común de cambio. En este espacio, compuesto por subjetividades de cada uno de los actores se abren posibilidades de acción a todos quienes activamente deseen participar, poniendo en agenda aquellas problemáticas que por diversas circunstancias no habían sido abordadas. En este sentido, no solo se constituyen en ideas de cambio, sino que se materializan en aspectos vinculados a medidas de organización y participación, incluso más allá de los límites del edificio de la escuela.

Como lo señala Zuluaga (2010) el docente desde la IAT se interesa en las estructuras de las prácticas sociales, reflejada por la vida cotidiana, la cultura barrial, tradiciones, etc., en su calidad de expresiones simbólicas que implican re-constituir una conciencia popular orientada a lograr el desarrollo humano.

\section{3. “Competitividad v/s Colaboración": Trabajo Colaborativo y Co-Agencia.}

El desarrollo profesional docente se ha vuelto una prioridad en el contexto de una sociedad neoliberal basada en el valor de la competencia como influjo principal para la mejora. Pero también resulta factible generar procesos de formación continua que fomenten la colaboración y el desarrollo profesional. El aprendizaje es especialmente efectivo cuando se realiza compartiéndolo con otros (Marqués, 2003), el grupo construye su aprendizaje creando en colaboración una cultura en la que se comparten contenidos y significados. Fomentar culturas de colaboración y de agenciamiento colectivo, implica aprender continuamente a múltiples niveles y tiene un peso innegable en el desarrollo profesional.

El planteamiento de este artículo es que la IAT hace posible formar la mirada a través una lógica donde la práctica docente cotidiana puede ser entendida desde la construcción cooperativa. El objetivo sería fomentar instancias de análisis, espacios conversacionales en los que se examinen las fracturas de nuestros sistemas educativos $\mathrm{y}$, desde ese lugar, anticipar respuestas contextualizadas, solidarias y pertinentes a las necesidades de aprendizaje que presentan los estudiantes. Todo ello desde una perspectiva que busque llevar a la acción valores como la equidad, la participación y la comunidad (Booth \& Ainscow, 2012).

La creación de culturas colaborativas en las instituciones educativas se convierte en un anhelo compuesto de grandes desafíos vinculados con el despliegue de una escuela abierta a la pluralidad y diversidad. La necesidad de llevar a cabo procesos de investigación en el contexto educativo se vincula con la propuesta de generar conversaciones que superen aquellas barreras que generalmente caracterizan al ámbito escolar.

\section{4. "Reproducción v/s Transformación". Nociones de Transformabilidad y Cambio.}

En la medida que el desarrollo profesional se aleje de la vivencia docente, de la experiencia de aula y de su problematización, para posicionarse en marcos de referencia meramente academicistas y reproductivos, se establece una desconexión con la necesidad de cambio y su significado profundo. Sin una debida atención a la cultura docente, las propuestas de desarrollo profesional no tendrán el impacto transformacional que nuestra educación necesita. Es entonces donde vinculamos el anhelado desarrollo profesional con este rol docente investigador, crítico y transformador, levantando dispositivos que actúen como dinamizadores de nuevas culturas y propiciando aprendizajes en un contexto de convivencia plural e inclusiva.

La visualización de los problemas de aprendizaje implica para el profesorado reconocer que existe una situación actual reflejada en el comportamiento manifiesto de los estudiantes, pero también que existen procesos de agenciamiento del propio educador que inciden en la no consecución de resultados de aprendizaje. Es por ello que la espiral reflexión-acción-reflexión permite buscar alternativas contextualizadas que vinculen explícitamente la enseñanza al aprendizaje. La iniciativa para emprender un proceso como éste, se da en gran parte porque se cree que es posible transformar o mejorar aquella situación inicial hacia otra mejor, más compleja, porque se tiene la convicción de que sólo de esta manera se logrará llegar a mejorar la calidad y profundidad de los aprendizajes desarrollados. 
En este sentido, hacemos notar la coherencia con el concepto de Transformabilidad propuesto por el movimiento "Aprendizaje Sin Límites" (Hart, Dixon, Drummond \& Mcintyre, 2004), el que refiere al conjunto de representaciones que levantan los y las docentes acerca de la capacidad de aprender de todos los estudiantes, la cual puede cambiar y ser mejorada como resultado de lo que el docente puede hacer en el presente. Esta visión pone de manifiesto una clara interdependencia entre los procesos de enseñanza y aprendizaje, y enarbola una fuerte crítica al etiquetaje de habilidades y a las perspectivas tradicionales y rígidas sobre los procesos de enseñanza.

\section{5. "Estandarización v/s Diversificación". Desarrollo profesional docente contextualizado y diversificado.}

Imbernón (2002) plantea como necesario el hecho de vincular el aprendizaje y la formación continua docente con su desarrollo profesional. Para esto se debe considerar la diversidad del profesorado y de sus territorios, contextualizar el desarrollo profesional y favorecer el análisis de las finalidades, contenidos y metódicas de la formación, a fin de lograr situarse en la práctica docente, favoreciendo en todo momento la pertinencia educativa y la profesionalidad.

Las políticas de desarrollo profesional docente deben tomar en consideración dichos tópicos, ya que el principal énfasis de la formación de profesores radica en los contenidos de aprendizaje, principalmente debido al influjo de las evaluaciones estandarizadas que miden lo que estrechamente el modelo educativo neoliberal considera como "calidad". Así pues, resulta urgente establecer un proceso de aprendizaje continuo que contemple las características de los propios docentes, sus contextos educativos y que aborde sus necesidades de formación a partir de la reflexión situada, capaz de proponer autónomamente cambios en su práctica docente.

Para romper con la racionalidad técnica del quehacer docente, esta formación debiera ser en esencia problematizadora. Para ello los/as propios/as docentes que ofician como aprendices, en vez de actuar como meros receptores de los contenidos de sus capacitaciones (entendidos por el autor como depósitos cognoscitivos) deben transformarse progresivamente en investigadores críticos (Freire, 1975 en Alcalá, 1998). Por lo mismo, la mirada de la formación continua debe privilegiar el desarrollo pleno de las capacidades de los y las docentes, potenciando sus conocimientos y ampliando sus competencias profesionales, pudiendo también reorientarlas para así abordar progresivamente sus propias necesidades y las de la sociedad (Bausela, 2009).

Para propiciar estos procesos renovadores del clásico "perfeccionamiento", las propuestas deben dejar de ser aisladas de la práctica, de tal modo que ésta se vuelva a integrar a la escuela como señalan los enfoques de reflexión sobre la práctica (Gómez \& Guerra, 2012). Favorecer una cultura de la autoevaluación y de reflexión sobre la práctica, implica favorecer una cultura más inclusiva y abierta a la diferencia (Figueroa \& Muñoz, 2014). Esto en sí mismo debiera implicar en el profesorado el cultivo de un "pensamiento ecologizante" (Zuluaga, 2010) que sitúe todo acontecimiento, información o conocimiento en una relación inseparable con su entorno. Para ello, es indispensable propiciar en nuestras escuelas contextos para que esto ocurra, mediando mejoras significativas en términos de condiciones laborales, propiciando tiempos adecuados de planificación de aula. Este tipo de condiciones puede permitir abordar desafíos tales como la co-enseñanza y la diversificación curricular a través de un enfoque que propicie acciones investigativas que problematicen y den cuenta de la complejidad de la práctica docente y de la vivencia del aula.

\section{Conclusiones}

Plantear un cambio implica creer que las transformaciones son posibles; se asumen riesgos que se podrían entender en la sociedad actual como los peligros que los seres humanos somos capaces de afrontar y que nos movilizan a evaluar y participar activamente del contexto del cual somos parte (Giddens 1998 como se citó en Del Moral \& Pita, 2002). Desde esta perspectiva, se busca proyectar un trabajo basado en la posibilidad de extender este proceso no sólo como un ideal formativo, sino como un objetivo alcanzable y concreto. También se pone de manifiesto la necesidad de docentes con inquietudes frente a lo que sucede tanto dentro como fuera del aula, posicionados en su quehacer y con intenciones compartidas desde la necesidad de aprender, adquiriendo conocimientos de otros y, además, generándolos desde su particular realidad. Se trata, en definitiva, de generar procesos de diálogo que tejan una verdadera red de aprendizajes entre profesores.

Es necesario reposicionar la reflexión como una práctica legítima dentro de nuestro sistema educativo, 
entendiendo que es necesario plantearla como un desafío para las políticas de desarrollo profesional docente. La reflexión estructura una participación activa, que moviliza y rescata aquellas preguntas (muchas veces proscritas) que se encuentran sumergidas en miles de urgencias propias de la cotidianidad de la escuela y que, si bien son parte de la historia de cada establecimiento, desvían la atención de aquellos procesos que permitirían re-crear soluciones que los mismos docentes expresan en distintas instancias de encuentro cuando existe un otro que abre la posibilidad de dialogar. El desafío de democratizar la enseñanza y los procesos de investigación parte por empoderar a los agentes educativos en relación con estas temáticas. Promover el análisis crítico y la autoconsciencia claramente nos sitúa con un mayor potencial para transformar los sistemas educativos.

Las escuelas deben potenciarse como lugares de aprendizaje profesional, en ese sentido la recuperación del itinerario seguido por experiencias, como por ejemplo los Microcentros rurales o las Comunidades de aprendizaje (Junji e Integra), pasa a ser una tarea casi obligatoria. El único modo de abordar los desafíos de una carrera docente a la altura de una visión de la educación como instrumento de cohesión social y desarrollo humano es reconocer el valor de lo dialógico, plural y culturalmente situado. Es en este punto donde la visión de comunidad, la confianza y la co-agencia (Hart et. al., 2004) pasan a ser componentes fundamentales en términos del contenido y la forma de la enseñanza. Si nuestro anhelo es formar ciudadanos críticos que aprendan a vivir en diversidad y comunidad, debemos comprender la necesidad urgente de abordar un desarrollo profesional docente en concordancia, como itinerario de mejora.

El ser conscientes y críticos del panorama global de la educación actual se torna relevante en contextos de diálogo permanente entre teoría y práctica. Esto porque el potencial de aprendizaje se maximiza en contacto con otros, por lo que resulta necesario nutrirse de procesos reflexivos que vuelvan cíclicamente a la práctica, transformando nuestros sistemas desde un horizonte de equidad y justicia social. Asimismo resulta fundamental el hecho de no restringir el conocimiento a procesos de indagación tradicionales, sino potenciar un conocimiento que surja desde la experiencia de los sujetos como eje central de su historia. Esto requiere no sólo una transformación explicitada en acciones concretas de gestión, planificación, reflexión, análisis de interacciones o resultados visibles públicamente sino también acceder a aquellas percepciones, concepciones y creencias implícitas que no sólo se visualizan en el profesorado sino también en todos aquellos que se hacen parte de reflexiones en torno a la educación.

En este contexto, es necesario propiciar espacios de desarrollo profesional docente que fomenten el abordaje de las disputas paradigmáticas, en términos de racionalidades subyacentes al acto educativo, desde el diálogo y la aceptación del otro, siempre propiciando un combate férreo al status-quo y a la educación reproductiva. Potenciando la función docente, sus condiciones laborales y su profesionalidad, se potenciará nuestra educación en tanto herramienta de desarrollo humano, a partir de los principios de equidad, justicia y democracia. Procesos de reforma como los que vivimos hoy en día se transforman en oportunidad para la agencia de propuestas alternativas a las lógicas imperantes. Para esto debemos disponer estrategias que rompan el monopolio del saber, generando desde la innovación y el empoderamiento local, las condiciones necesarias para el desarrollo de la autonomía profesional y la autodeterminación docente.

\section{Referencias}

Alcalá, A. (1997). Propuesta de Una Definición Unificadora de Andragogía [Documento de trabajo]. Recuperado de http://postgrado. una.edu.ve/andragogia2007-2/paginas/alcala1997propuesta.pdf

Amaya-De Rebolledo, R \& Amaya, Z. (2010). Mediación de la cultura docente en los proyectos de formación permanente. $M a$ gis, Revista Internacional de Investigación en Educación, 3(5), 135-146. Recuperado de http://revistas.javeriana.edu.co/index. php/MAGIS/article/view/3531

Arias, C. \& Restrepo, M. (2009). La investigación-acción en educación: un camino hacia el desarrollo profesional y la autonomía. Revista Ikala de Lenguaje y Cultura, 14(22), 109-122. Recuperado de http://ref.scielo.org/69byx 7

Ávalos, B. (2007). El desarrollo profesional continuo de los y las docentes: Lo que nos dice la experiencia internacional y de la región latinoamericana. Revista Pensamiento Educativo, 41(2), 77-99. Recuperado de http://pensamientoeducativo.uc.cl/index. $\mathrm{php} / \mathrm{pel} /$ article/view/417

Bausela, E. (2009). La Docencia a través de la Investigación Acción. Revista Iberoamericana de Educación, 20, 7-36. Recuperado de http://www.rieoei.org/deloslectores/682Bausela.PDF

Baquero, R. (2004). Vygotski y el aprendizaje Escolar. Buenos Aires: AIQUE.

Barber, M. \& Mourshed, M. (2008). Cómo hicieron los sistemas educativos con mejor desempeño del mundo para alcanzar sus objetivos. McKinsey \& Company: PREAL.

Beca, C. (2005). Acciones de Desarrollo profesional en Chile. Encuentro Internacional: Los Desafíos de la Formación en la Sociedad del Conocimiento, Santiago, Chile.

Blandez, J., González, B., López, A. \& Sierra, M. (2006). La Formación de Profesores Responsables a través de la Investigación Acción. Revista de Investigación Educativa, 24(1), 71-95. Recuperado de http://revistas.um.es/rie/article/view/97301 
Boggino, N. \& Rosekrans, K. (2007). Investigación acción: reflexión crítica sobre la práctica educativa, orientaciones prácticas y experiencias. Madrid: Homosapiens.

Booth, T. \& Ainscow, M. (2012). La Guía para la Inclusión Educativa. Santiago: Fundación Creando Futuro.

Castorina J. \& Dubrovsky, S. (2004). La enseñanza y la teoría Psicológica socio-histórica. Algunos problemas conceptuales. En J. A. Castorina \& S. Dubrobsky (Eds.), Psicología, Cultura y Educación, perspectivas desde la obra de Vigotsky (pp. 81-104). Buenos Aires: Noveduc.

Centro de Investigación y Desarrollo de la Educación, Universidad Alberto Hurtado (2012). Procesos de Enseñanza y Aprendizaje desde la perspectiva de los profesores de Chile (Informe presentado por: Universidad Alberto Hurtado/Facultad de Educación/Centro de Investigación y Desarrollo de la Educación CIDE a UNESCO). Recuperado de http://www.cide.cl/documentos/Procesos_de_ensenanza_aprendizaje_desde_la_perspectiva_de los_profesores.pdf

Darling-Hammond, L. (2003). Keeping Good Teachers: Why it Matters, What Leaders Can Do. Educational leadership, 60(8), 6-13. Recuperado de http://www.ascd.org/publications/educationalleadership/may03/vol60/num08/Keeping-Good-Teachers@-WhyIt-Matters,-What-Leaders-Can-Do.aspx

Del Moral, L. \& Pita, M. (2002). El papel de los riesgos en las sociedades contemporáneas. En A. Carcedo \& O. Cantos (Eds.), Riesgos naturales (pp. 75-88). Barcelona: Ariel.

Duhalde, M. \& Cardelli, J. (2001). Formación docente en América Latina. Una perspectiva político-pedagógica. Cuadernos de Pedagogía, 308, 38-45. Recuperado de http://www.oei.es/ docentes/articulos/formacion_docente_AL_perspectiva_politico_pedagogica.pdf

Elboj, C., Puigdellívol, I., Soler, M. \& Valls, R. (2002). Comunidades de Aprendizaje: transformar la educación. Barcelona: Graó.

Elliott, J. (2000). La investigación-acción en educación. Madrid: Morata.

Escudero, J. (2009). Comunidades docentes de aprendizaje, formación del profesorado y mejora de la educación. Revista Ágora para la EF y el Deporte, (10), 7-31. Recuperado de https://www5.uva. es/agora/revista/10/agora10_escudero.pdf

Fernández Enguita, M. (1995). La escuela a Examen. Madrid: Pirámide.

Figueroa, I. \& Muñoz, Y. (2014). La Guía para la Inclusión Educativa como herramienta de autoevaluación institucional: Reporte de una experiencia. Revista Latinoamericana de Educación Inclusiva, 8(2), 179-198. Recuperado de http://www.rinace.net/rlei/numeros/ vol8-num2/art9.pdf

Fondo de las Naciones Unidas para la Infancia. (2004). ¿Quién Dijo que no se puede? Escuelas efectivas en sectores de pobreza. Santiago: MINEDUC.

Freire, P. (1993). Pedagogía de la esperanza. México: Siglo XXI.

García-Huidobro, J. (2007). Desigualdad educativa y segmentación del sistema escolar. Revista Pensamiento Educativo, 40(1), 65-85. Recuperado de http://pensamientoeducativo.uc.cl/index.php/pel/ article/view/399

Giroux, H. (1997). Los profesores como intelectuales: hacia una pedagogía crítica del aprendizaje. Barcelona: Paidós.

Gómez, V. \& Guerra, P. (2012). Teorías implícitas respecto a la enseñanza y el aprendizaje: ¿Existen diferencias entre profesores en ejercicio y estudiantes de pedagogía?. Estudios Pedagógicos, 38(1), 25-43. doi:10.4067/S0718-07052012000100001

Hart, S., Dixon, A., Drummond, M. \& Mcintyre, D. (2004). Learning without Limits. New York: Open University Press.

Herrera, J. (27 de Junio de 2015). Las mejores y peores áreas pedagógicas de los profesores. La Tercera. Recuperado de http:// www.latercera.com/noticia/nacional/2015/06/680-636290-9-lasmejores-y-peores-areas-pedagogicas-de-los-profesores.shtml
Hernández, P., Fernández, C. \& Baptista, L. (2008). Metodología de la Investigación. México, D.F: McGraw-Hill Interamericana.

Imbernón, F. (2001). Claves para una Nueva Formación del Profesorado. Revista Investigación en la Escuela. 43, 57-66. Recuperado de http://www.ub.edu/obipd/claves-para-una-nueva-formaciondel-profesorado/

Imbernón, F. (2002). Reflexiones globales sobre la formación y el desarrollo profesional del profesorado en el estado español y latinoamericano. Revista Educar, 30, 15-25. Recuperado de http:// www.raco.cat/index.php/Educar/article/view/20761

Ivic, I. (1994). Lev Semionovich Vygostky (1896-1934). Revista Perspectivas, 24(3), 773-799. Recuperado de http://www.ibe. unesco.org/International/Publications/Thinkers/ThinkersPdf/ vygotskys.PDF

Latorre, A. (2003). La Investigación-Acción. Conocer y cambiar la práctica educativa. Barcelona: Graó.

Molina Ruiz, E. (2003). Creación y desarrollo de Comunidades de Aprendizaje. Hacia la Mejora Educativa. Revista de Educación, (337), 235-250. Recuperado de http://www.revistaeducacion.mec. es/re337_12.htm

Martinic, S., \& Vergara, C. (2007). Gestión del tiempo e interacción del profesor-alumno en la sala de clases de establecimientos de Jornada Escolar Completa en Chile. Revista Electrónica Iberoamericana sobre Calidad, Eficacia y Cambio en Educación, 5(5), 3-20. Recuperado de http://www.rinace.net/arts/vol5num5e/art1.htm

Ministerio de Educación (2002). Política de convivencia escolar. Santiago: MINEDUC.

Ministerio de Educación (2008). Marco para la buena enseñanza. Santiago: MINEDUC.

OPECH (2007). Tensiones de la profesión docente y sus políticas de Desarrollo [Documento de trabajo]. Recuperado dehttp:// www.opech.cl/inv/documentos_trabajo/Doc_Trab05_Tensiones_de_la_profesion_Docente.pdf

Parica, A., Bruno, F. \& Abancin, R. (2005). Teoría del Constructivismo Social de Vygotski en Comparación con la Teoría de Jean Piaget [Mensaje en un blog]. Recuperado de http://constructivismos. blogspot.cl/2005/06/teoria-del-constructivismo-social-de.html

Pérez Echeverría, M., Mateos, M., Scheuer, N. \& Martín, E. (2006). Enfoques en el estudio de las concepciones sobre el aprendizaje y la enseñanza. En J. I. Pozo, N. Scheuer, M. Pérez Echeverría, M. Mateos, E. Martín, \& M. De la Cruz (Eds.), Nuevas formas de pensar la enseñanza y el aprendizaje. Las concepciones de profesores y alumnos (pp. 55-94). Barcelona: Graó

Samaras, A. \& Freese, A. (2009). Looking Back and Looking Forward: An Historical Overview of the Self Study Research Methodologies for Teacher Educators. En C. A. Lassonde, S. Galman \& C. Kosnik (Eds.), Self-Study Research Methodologies for Teacher Educators (pp. 3-19). Rotterdam: Sense Publishers.

Santos, M. (2000). Exordio para ciudadanos críticos. En M. A. Santos (Ed.), La escuela que Aprende (pp. 11-22). Madrid: Morata.

Strauss, A. \& Corbin, J. (2002). Bases de la Investigación Cualitativa. Técnicas y Procedimientos para Desarrollar la Teoría Fundamentada. Colombia: Universidad de Antioquia.

Toomey, R. (1997). Transformative action research. Educational Action Research, 5(1), 105-121. doi:10.1080/09650799700200022

Torrado J. \& Pozo J. (2006). Del dicho al Hecho: de las concepciones sobre el aprendizaje a la práctica de la enseñanza de la música. En J. I. Pozo J. I., N. Scheuer, M. Pérez Echeverría M., M. Mateos M., E. Martín E., \& M. De la Cruz M., (Eds.) Nuevas formas de pensar la enseñanza y el aprendizaje. Las concepciones de profesores y alumnos (pp. 205-228). Barcelona: Graó.

Venegas P., Campos J., Cortés C. \& Novoa X. (2010). Las Comunidades de Aprendizaje, una estrategia de participación y desarrollo profesional en Chile, las desigualdades educativas en América latina. Serie ensayos e investigaciones, (1), 15-30. 
Vygotski, L. (1979). El desarrollo de los procesos psíquicos superiores. Barcelona: Edit. Crítica.

Zuluaga, R. (2010). La investigación acción transformadora: una apuesta para generar conocimiento articulando práctica docente y lo colectivo popular. Recuperado de http://www.pensamientocomplejo.com.ar/docs/files/Rocio Zuluaga de Prato, Investigacion Accion Transformadora .pdf 